Original Paper http://ajol.info/index.php/ijbcs http://indexmedicus.afro.who.int

\title{
Profil lipidique des personnes vivant avec le VIH sous antirétroviral suivis au Centre Hospitalier Universitaire Ouémé-Plateaux (CHU-O/P) de Porto-Novo, Bénin
}

Adébayo ALASSANI ${ }^{1 *}$, Albert Comlan DOVONOU ${ }^{1}$, Angelo Cossi ATTINSOUNON ${ }^{1}$, Jules GNINKOUN ${ }^{3}$, Moutawakilou GOMINA ${ }^{1}$, Armand WANVOEGBE ${ }^{4}$, Léopold CODJO $^{1}$, AZANDJEME Colette ${ }^{5}$, Djimon Marcel ZANNOU ${ }^{3}$ et François DJROLO ${ }^{3}$

\author{
${ }^{I}$ Centre Hospitalier Universitaire Départemental du Borgou-Alibori, Parakou, Bénin \\ ${ }^{2}$ Faculté des Sciences de Santé, Cotonou, Bénin. \\ ${ }^{3}$ Centre National Hospitalier Universitaire Hubert Koutoucou Maga, Cotonou, Bénin. \\ ${ }^{4}$ Centre Hospitalier Universitaire de l'Ouémé-Plateau, Porto-Novo, Bénin. \\ ${ }^{5}$ Institut Régional de Santé Publique, Ouidah, Bénin. \\ *Auteur correspondant; E-mail : adebayoalassani@gmail.com ; alsaco2007@yahoo.fr, Tél :0022995848436.
}

\section{RESUME}

Au regard des connaissances actuelles, aucune étude n'est disponible au Bénin sur le profil lipidique des personnes vivant avec le VIH. L'objectif de la présente étude est de décrire le profil lipidique des personnes vivant avec le VIH sous antirétroviral suivies au CHU-O/P de Porto-Novo. Il s'est agi d'une étude transversale, descriptive et analytique. La population d'étude a été constituée les personnes vivant avec le VIH sous antirétroviral suivies CHU-O/P de Porto-Novo. La collecte des données a été faite à la base d'une fiche de collecte chez les personnes éligibles et ayant donné leur consentement. Le profil lipidique a été apprécié par le bilan lipidique réalisé chez les patients à jeun. Au total 244 patients ont été inclus dans l'étude. On a noté une prédominance des femmes (74,6\%). La moyenne d'âge était de 40,7 $\pm 9,71$ ans. L'hypercholestérolémie totale a été observée chez 101 patients (41,39\%), l'hypercholestérolémie LDL chez 84 patients (34,83\%), l'hypocholestérolémie HDL chez 68 patients $(27,87 \%)$ et l'hypertriglycéridémie chez 44 patients $(18,03 \%)$. Le rapport triglycéridémie sur cholestérolémie HDL est élevé chez 45 patients $(18,44 \%)$. Le surpoids a été associé aux dyslipidémies. Le profil lipidique des patients infectés par le VIH et sous antirétroviral de la présente étude est caractérisé par la présence des dyslipidémies favorisées par le surpoids.

(C) 2016 International Formulae Group. All rights reserved.

Mots clés: Profil lipidique, PVVIH, Porto-Novo, Bénin.

\section{Lipid profile of people living with HIV on antiretroviral monitored University Hospital Center Ouémé Plateaux (UHC-O/P) in Porto-Novo, Benin}




\section{ABSTRACT}

Given current knowledge, no study is available in Benin on the lipid profile of people living with HIV. The objective of this study is to describe the lipid profile of people living with HIV on antiretroviral followed in UHC-O/P of Porto-Novo. He acted in a cross-sectional study, descriptive and analytical. The study population was made up of people living with HIV antiretroviral followed in UHC-O/P of Porto-Novo. Data collection was made the basis of a collection sheet among people eligible and consenting. The lipid profile was appreciated by the lipid profile performed in fasted patients. In total 244 patients were included in the study. There was a predominance of women $(74.6 \%)$. The average age was $40.7 \pm 9.71$ years. The high total cholesterol was observed in 101 patients (41.39\%), high LDL cholesterol in 84 patients (34.83\%), low cholesterolemia HDL in 68 patients $(27.87 \%)$ and high triglyceridemia in 44 patients $(18.03 \%)$. The ratio triglycerides and HDL cholesterol is elevated in 45 patients $(18.44 \%)$. Overweight was associated with dyslipidemia. The lipid profile of patients infected with HIV and on antiretroviral this study is characterized by the presence of dyslipidemia favored by overweight.

(C) 2016 International Formulae Group. All rights reserved.

Keywords: Lipid profile, PLWHA, Porto-Novo, Benin.

\section{INTRODUCTION}

Le traitement antirétroviral très actif favorise une diminution considérable de l'incidence des affections opportunistes et une réduction de la mortalité liée au VIH (Belay et al., 2014) même si encore 1,3 millions de décès ont été enregistrées en 2013 (Tanjong et al., 2016). Les sujets infectés ont de plus en plus une augmentation de l'espérance de vie et une amélioration de la qualité de vie liées à l'utilisation des antirétroviraux (Berhane et al., 2013). Cependant, malgré les effets bénéfiques du traitement antirétroviral, on assiste à l'apparition des anomalies métaboliques; parmi lesquelles les dyslipidémies (Kouakou-Siransy et al., 2015). Elles sont caractérisées par une augmentation de la cholestérolémie totale, de la cholestérolémie LDL, de la triglycéridémie et une diminution de la cholestérolémie HDL (Habtamu et al., 2014 ; Marino et al., 2015). La pathogénie des dyslipidémies chez les personnes vivant avec le VIH sous antirétroviral n'est pas encore clairement définie. Plusieurs facteurs associés aux dyslipidémies ont été décrits. Parmi ces facteurs on cite les antirétroviraux, le VIH, les facteurs génétiques, le mode de vie des patients, les facteurs environnementaux (da Cunha et al., 2015; Lake et al., 2013; Bekolo et al., 2015). Les conséquences des dyslipidémies sur le risque cardio-vasculaires sont connues et diversement étudiées (Shahbaz et al., 2015; Bekolo et al., 2014; Ijeh et al., 2010). Au Bénin, des études se sont intéressées déjà aux anomalies métaboliques chez les personnes vivant avec le VIH sous antirétroviraux. Les anomalies métaboliques comme les lipodystrophies (Alassani et al., 2016) et le syndrome métabolique (Alassani et al., 2015) chez les sujets infectés par le VIH ont été étudiées. Cependant, aucune étude ne s'est intéressée au profil lipidique des personnes infectées par le VIH sous antirétroviraux. La présente étude a pour but d'apprécier le profil lipidique des ces patients afin de déterminer l'ampleur en vue d'une meilleure prise en charge globale.

\section{MATERIEL ET METHODES}

Type d'étude, population d'étude et critères d'éligibilité

Il s'est agi d'une étude transversale, descriptive et analytique. La population d'étude était constituée des personnes vivant avec le VIH sous antirétroviral suivies au Centre Hospitalier Universitaire de l'OuéméPlateau (CHU-O/P). Les patients non hospitalisés, les femmes non enceintes, ne prenant pas d'hypolipémiants et ayant donné 
leur consentement écrit ont été inclus dans l'étude.

\section{Collecte des données}

Une fiche de collecte des données a été élaborée et administrée à tous les patients inclus dans l'étude. Les patients ont été préalablement informés des modalités du déroulement de la collecte des données. La première phase a consisté au recueil des informations relatives au sexe, à l'âge, à la consommation de l'alcool ou du tabac, au niveau d'activité physique et au rappel alimentaire des 72 h. A la deuxième phase, les mesures anthropométriques comme le poids, la taille et le tour de taille ont été prises ainsi que la tension artérielle après un repos de 10 minutes. La durée du traitement et le type de traitement antirétroviral ont été obtenus dans le dossier individuel de suivi des patients. La dernière phase a consisté au prélèvement sanguin chez les patients préalablement à jeun pour la réalisation du bilan lipidique (Cholestérolémie totale, Cholestérolémie HDL, Triglycéridémie) et de la glycémie. Le taux de la cholestérolémie LDL a été calculé par la formule de Friedewald.

\section{Définition des variables}

La présence d'une anomalie au niveau du bilan lipidique a été qualifiée de dyslipidémie selon les critères de National Cholesterol Education Program, Adult Treatment Panel III qui sont : hypercholestérolémie totale (HCT) si cholestérolémie totale supérieure ou égale à 2 g/L, hypertriglycéridémie (HTG) si triglycéridémie supérieure ou égale à $1,5 \mathrm{~g} / \mathrm{L}$, hypocholestérolémie HDL $(\mathrm{HCH})$ si cholestérolémie HDL inférieure à $0,5 \mathrm{~g} / \mathrm{L}$ chez la femme et inférieure à $0,4 \mathrm{~g} / \mathrm{L}$ chez l'homme, hypercholestérolémie LDL (HCL) si cholestérolémie LDL supérieure ou égale à 1,3 g/L et le rapport triglycéridémie sur cholestérolémie HDL élevé si supérieur ou égal à 5. Le tour de taille élevé a été défini selon les critères de la Fédération Internationale du Diabète (tour de taille supérieur ou égal à $94 \mathrm{~cm}$ chez l'homme et supérieur à $80 \mathrm{~cm}$ chez la femme). La sédentarité a été définie pour une durée de marche rapide par jour inférieure à 30 minutes.

\section{Analyses statistiques}

L'analyse des données a été faite par les logiciels Alimenthèque pour l'appréciation des apports énergétique et lipidique et Statistical Package for Social Sciences (SPSS) version 20.0. pour les autres variables. Le test de Chi carré a été calculé et une p-value inférieure à 0,05 a été considérée comme significative.

\section{Considérations éthiques}

L'autorisation du Directeur CHU-O/P, du responsable du Centre de Traitement Ambulatoire des personnes infectées par le VIH et du Président des personnes vivant avec le VIH suivies dans le centre a été obtenue. Le consentement écrit des patients a été obtenu. L'anonymat et la confidentialité des résultats ont été respectés. Les prélèvements pour le bilan lipidique et la glycémie sont habituellement faits au contrôle des patients infectés par le VIH. Les patients ont été informés des modalités de l'étude.

\section{RESULTATS}

\section{Caractéristiques générales de la population d'étude}

Au total 252 patients ont été recensés parmi lesquels 4 hommes sous statine et 4 femmes enceintes ont été exclus de l'étude. Parmi les 244 patients qui ont été inclus dans l'étude, on a noté une prédominance des femmes $(74,6 \%)$. La moyenne d'âge était de 40,7 \pm 9,71 ans avec une prédominance des patients âgés de moins de 40 ans $(57,4 \%)$. L'hyperglycémie a été observée chez 25 patients $(9,76 \%)$. Seulement 5 patients $(2,04 \%)$ ont été diabétiques. Le surpoids a été observé chez 75 patients $(30,74 \%)$. Le tour de 
taille élevé a été observé chez 125 patients (51,23\%). L'hypertension artérielle a été diagnostiquée chez 60 patients $(24,59 \%)$. Près de la moitié des patients $(49,60 \%)$ est sédentaire. L'apport énergétique est élevé chez 63 patients $(25,82 \%)$ Tableau 1.

\section{Profil lipidique des patients}

L'hypercholestérolémie totale a été observée chez 101 patients (41,39\%), l'hypercholestérolémie LDL chez 84 patients (34,83\%), l'hypocholestérolémie HDL chez 68 patients $(27,87 \%)$ et l'hypertriglycéridémie chez 44 patients $(18,03 \%)$. Le rapport
Triglycéridémie sur cholestérolémie HDL est élevé chez 45 patients $(18,44 \%)$ Tableau 2.

La moyenne de la cholestérolémie totale était de $1,90 \pm 0,37 \mathrm{gL}^{-1}$, celle de la cholestérolémie LDL $1,06 \pm 0,31 \mathrm{gL}^{-1}$, celle de la cholestérolémie HDL $0,66 \pm 0,14 \mathrm{gL}^{-1}$ et celle de la triglycéridémie $0,85 \pm 0,25 \mathrm{gL}^{-1}$.

Le surpoids a été significativement associé à l'hypercholestérolémie totale, hypercholestérolémie LDL, l'hypocholestérolémie HDL et l'hypertriglycéridémie. Aucune relation n'a été observée entre le surpoids et la durée du traitement et la durée de traitement et le profil lipidique (Tableaux 3 et 4).

Tableau 1 : Caractéristiques générales de la population d'étude des personnes infectées par le VIH sous antirétroviral suivies au CHD-O/P $(\mathrm{n}=244)$.

\begin{tabular}{|c|c|c|c|}
\hline Variables & $\mathrm{n}(\%)$ & Variables & $\mathbf{n}(\%)$ \\
\hline Sexe & & Age & \\
\hline Féminin & $179(73,36)$ & Age $<40$ ans & $140(57,37)$ \\
\hline Masculin & $65(26,64)$ & Age $\geq 40$ ans & $104(42,63)$ \\
\hline \multicolumn{2}{|c|}{ Indice de masse corporelle } & \multicolumn{2}{|c|}{ Durée du traitement antirétroviral } \\
\hline Pas de surpoids & $169(69,26)$ & $<1$ an & $6(2,46)$ \\
\hline Surpoids & $75(30,74)$ & $\geq 1$ an & $238(97,54)$ \\
\hline \multicolumn{2}{|c|}{ Schéma thérapeutique } & \multicolumn{2}{|c|}{ Apport énergétique } \\
\hline $2 \mathrm{INTI}+\mathrm{INNTI}$ & $239(97,95)$ & Bas ou normal & $181(74,18)$ \\
\hline $2 \mathrm{INTI}+\mathrm{IP}$ & $5(2,05)$ & Elevé & $63(25,82)$ \\
\hline \multicolumn{2}{|l|}{ Apport lipidique } & \multicolumn{2}{|c|}{ Consommation d'alcool } \\
\hline Normal & $102(41,80)$ & Oui & $112(45,90)$ \\
\hline Elevé & $142(58,20)$ & Non & $132(54,10)$ \\
\hline \multicolumn{2}{|c|}{ Consommation de tabac } & \multicolumn{2}{|c|}{ Niveau d'activité physique } \\
\hline Oui & 243(99,59) & Actif & $123(50,40)$ \\
\hline Non & $1(0,041)$ & Sédentaire & $121(49,60)$ \\
\hline \multicolumn{2}{|l|}{ Tour de taille } & \multicolumn{2}{|c|}{ Hypertension artérielle } \\
\hline Normal & $119(48,77)$ & Présente & $60(24,59)$ \\
\hline Elevé & $125(51,23)$ & Absente & $184(75,41)$ \\
\hline \multicolumn{2}{|l|}{ Hyperglycémie } & \multicolumn{2}{|l|}{ Diabète } \\
\hline Présente & $25(9,76)$ & Présent & $5(2,04)$ \\
\hline Absente & $219(90,24)$ & Absent & $239(97,96)$ \\
\hline
\end{tabular}


Tableau 2 : Profil lipidique des personnes infectées par le VIH sous antirétroviral suivies au CHD-O/P (n=244).

\begin{tabular}{lc}
\hline Variables & $\mathbf{n}(\%)$ \\
\hline HCT & $101(41,39)$ \\
Oui & $143(58,61)$ \\
Non & \\
HCL & $84(34,83)$ \\
Oui & $160(65,17)$ \\
Non & \\
HCH & $68(27,87)$ \\
Oui & $176(72,13)$ \\
Non & \\
HTG & $44(18,03)$ \\
Oui & $200(81,97)$ \\
Non & $199(81,56)$ \\
Rapport Triglycéridémie sur Cholestérolémie & HDL \\
Normal & $45(18,44)$ \\
Elevé &
\end{tabular}

Tableau 3 : Relation entre surpoids et durée du traitement antirétroviral.

\begin{tabular}{llll}
\hline & $\begin{array}{l}\text { Surpoids } \\
\mathbf{n}(\%)\end{array}$ & $\begin{array}{l}\text { Pas de surpoids } \\
\mathbf{n}(\%)\end{array}$ & $\mathbf{p}$ \\
\hline $\begin{array}{l}\text { Durée de traitement antirétroviral } \\
<1 \text { an }\end{array}$ & $2(33,33)$ & $4(66,67)$ & 0,8890 \\
$\geq 1$ an & $73(30,67)$ & $165(69,33)$ & \\
\hline
\end{tabular}

Tableau 4 : Relation entre surpoids, durée du traitement antirétroviral et profil lipidique.

\begin{tabular}{|c|c|c|c|c|c|c|c|c|}
\hline & $\begin{array}{l}\text { HCT } \\
\mathrm{n}(\%)\end{array}$ & $\mathrm{P}$ & $\begin{array}{l}\mathbf{H C L} \\
\mathrm{n}(\%)\end{array}$ & $\mathrm{p}$ & $\begin{array}{l}\mathbf{H C H} \\
\mathrm{n}(\%)\end{array}$ & $\mathrm{p}$ & $\begin{array}{l}\text { HTG } \\
\mathrm{n}(\%)\end{array}$ & $\mathrm{p}$ \\
\hline \multicolumn{9}{|c|}{ Surpoids } \\
\hline Oui & $65(86,7)$ & $<10^{-4}$ & $40(53,3)$ & $<10^{-4}$ & $43(57,3)$ & $<10^{-4}$ & $20(20,7)$ & 0,019 \\
\hline Non & $36(21,3)$ & & $44(26)$ & & $25(14,8)$ & & $24(14,2)$ & \\
\hline \multicolumn{9}{|c|}{ Durée de traitement } \\
\hline $\begin{array}{l}<1 \text { an } \\
\geq 1 \text { an }\end{array}$ & $\begin{array}{l}2(33,3) \\
99(41,6)\end{array}$ & 0,684 & $\begin{array}{l}1(16,7) \\
83(34,9)\end{array}$ & 0,353 & $\begin{array}{l}3(50) \\
65(27,9)\end{array}$ & 0,220 & $\begin{array}{l}2(33,3) \\
42(17,5)\end{array}$ & 0,323 \\
\hline
\end{tabular}

\section{DISCUSSION}

Le profil des patients infectés par le VIH sous antirétroviral est caractérisé par la présence des dyslipidémies. L'hypercholestérolémie totale a été observée chez $41,39 \%$ des patients, l'hypercholestérolémie LDL chez 34,83\%, l'hypocholestérolémie HDL chez $27,87 \%$ et l'hypertriglycéridémie chez $18,03 \%$. Plusieurs études ont rapporté un profil lipidique semblable. Abebe et al. (2014) et Belay et al. (2014) ont rapporté une prévalence de l'hypercholestérolémie semblable à la nôtre avec respectivement $42,1 \%$ et $45,7 \%$. Dans les études de Hejazi et al. (2013) et de de Limas et al. (2014), l'hypercholestérolémie LDL est observée chez respectivement $35,1 \%$ et $33,1 \%$. Les résultats des études de Nsagha et 
al. (2015) et Bekolo et al. (2014) confirment la faible prévalence de l'hypocholestérolémie HDL de la présente étude avec respectivement $14 \%$ et $18,4 \%$. Dans la même étude de Nsagha et al. (2015), une faible prévalence de l'hypertriglycéridémie a été rapportée; dans son étude seulement 1 patient sur $5(20,4 \%)$ ont une hypertriglycéridémie. Le rapport triglycéridémie sur cholestérolémie HDL élevé qui caractérise un risque d'athérogénicité élevé est observé chez $18,44 \%$ des patients est semblable à celui de $17,9 \%$ rapporté par Daniyam et al. (2013). Les patients infectés par le VIH de la présente ont des moyennes de la cholestérolémie totale, de la cholestérolémie HDL et de la triglycéridémie normales respectivement à $1,90 \pm 0,37 \quad \mathrm{gL}^{-1}, 0,66 \pm 0,14 \mathrm{gL}^{-1}$ et $0,85 \pm$ $0,25 \mathrm{gL}^{-1}$. La valeur de la cholestérolémie totale de cette étude a confirmé les résultats de Armstrong et al. (2011) et de Gowdaiah et al. (2015) qui ont rapporté respectivement une moyenne de la cholestérolémie totale à 1,42 $\mathrm{gL}^{-1}$ et $1,76 \mathrm{gL}^{-1}$. Dans l'étude de Oyong et al. (2016), une cholestérolémie HDL normale a été rapportée à $0,52 \mathrm{gL}^{-1}$. Il en est de même de la moyenne normale de la triglycéridémie à $1,17 \mathrm{gL}^{-1}$ rapportée Nsagha et al. (2015). Par contre, la moyenne de la cholestérolémie LDL est élevée à $1,06 \pm 0,31 \mathrm{gL}^{-1}$. Dans les études de Indumati et al. (2014) et de Nsagha et al. (2015), une cholestérolémie LDL élevée a été rapportée avec respectivement une moyenne de $1,27 \mathrm{gL}^{-1}$ et $1,17 \mathrm{gL}^{-1}$. Les caractéristiques socio-démographiques ont noté une prédominance féminine et la jeunesse de la population d'étude avec une moyenne d'âge de 40,7 $\pm 9,71$ ans. La prédominance féminine de la population d'étude a confirmé celle rapportée par Zhou et al. (2015) et Habtamu et al. (2014). Quant à la jeunesse de la population d'étude, Shen et al. (2015) et Lozès et al. (2012) ont rapporté des résultats similaires avec respectivement une moyenne d'âge de 36,34 ans et 38 ans.

Le surpoids a été associé aux dyslipidémies dans la présente étude. Habtamu et al. (2014) ont abouti à la même association entre le surpoids et les dyslipidémies. Dans l'étude de Abebe et al. (2014) la prévalence de l'hypercholestérolémie totale et de l'hypocholestérolémie HDL augmente avec un indice de masse corporelle. Pour prévenir donc les dyslipidémies, une lutte contre le surpoids s'avère nécessaire

\section{Conclusion}

Le profil lipidique des patients infectés sous antirétroviral de la présente étude est caractérisé par la présence des dyslipidémies. La lutte contre le surpoids permettra de prévenir les dyslipidémies et d'éviter les maladies cardio-vasculaires.

\section{CONFLIT D'INTERET : Aucun}

\section{CONTRIBUTION DES AUTEURS}

AA a conçu l'étude. DCA, AAC, GJ, GM et WA ont développé le protocole et ont recueilli les données sous la supervision de ZDM et DF, AA et CL ont analysé les données et écrit la première version du manuscrit. Tous les co-auteurs ont contribué à la révision et à la finalisation du document.

\section{REMERCIEMENTS}

Ils vont à l'endroit de toutes les personnes infectées par le VIH suivis au Centre Hospitalier Universitaire OuéméPlateaux et à toute l'équipe du Centre de Traitement Ambulatoire des personnes infectées par le VIH.

\section{REFERENCES}

Abebe M, Kinde S, Belay G, Gebreegziabxier A, Challa F, Gebeyehu T, Nigussie P, Tegbaru B. 2014. Antiretroviral treatment associated hyperglycemia and dyslipidemia among HIV infected patients at Burayu Health Center, Addis Ababa, Ethiopia: a cross-sectional comparative study. BMC Research Notes, 7: 380 .

Alassani A, Dovonou AC, Attinsounon AC, Gninkoun J, Wanvoegbe A, Codjo L, Zannou DM, Djrolo F, Houngbé F. 2016. Prevalence of lipodystrophy and 
associated factors in adults living with HIV on antiretroviral followed Bethesda Hospital in Cotonou in 2014. Revue Malienne d'Infectiologie et de Microbiologie, 7: 18-27.

Alassani A, Dovonou AC, Sossou E, Attinsounon AC, Gninkoun J, Wanvoegbe A, Ahoui S, Codjo L, Adè G. 2015. Prévalence, facteurs associés et prédisposant au syndrome métabolique chez les personnes vivants avec le VIH sous traitement antirétroviral à PortoNovo en 2014. PanAfrican Medical Journal, 22: 296.

Armstrong C, Liu E, Grinspoon S, Okuma J, Spiegelman D, Chalamilla G. 2011. Dyslipidemia in an HIV-positive, antiretroviral treatment-naïve population in Dar es Salaam, Tanzania. Acquir Immune Defic. Syndr., 57(2): 141-145.

Bekolo CE, Nguena MB, Ewane L, Bekoule PS, Kollo B. 2014. The lipid profile of HIV-infected patients receiving antiretroviral therapy in a rural Cameroonian population. BMC Public Health., 14: 236.

Belay E, Seifu D, Amogne W, Kibret KT. 2014. Lipid Profile Derangements among Human Immunodeficiency Virus Infected Adults Receiving First Line AntiRetroviral Therapy in Tikur Anbesa Specialized Hospital, Addis Ababa, Ethiopia: Comparative Cross-Sectional Study. J. AIDS Clin. Res., 5(8): 1-7.

Berhane T, Yami A, Alemseged F, Yemane T, Hamza L, Kassim M. 2012. Prevalence of lipodystrophy and metabolic syndrome among HIV positive individuals on Highly Active Anti-Retroviral treatment in Jimma, South West Ethiopia. Pan African Medical Journal, 13: 43.

da Cunha J, Maselli L, Stern A, Spada C, Bydlowki SP. 2015. Impact of antiretroviral therapy on lipid metabolism of human immunodeficiency virusinfected patients: Old and new drugs. World Journal of Virology, 4(2): 56-77.

Daniyam CA, Iroezindu MO. 2013. Lipid profil of anti-retroviral treatment-naïve
HIV-infected patients in Jos, Nigeria. Annals of Medical and health Sciences Research., 3(1): 26-31. DOI: http://www.ajol.info/index.php/amhsr/arti cle/viewFile/87716/77375

de Limas TG, Pinto G, Marcato LM, Coelho DR. 2014. Analysis of the prevalence of dyslipidemia in individuals with HIV and its association with antiretroviral therapy. Revista da Sociedade Brasileira de Medicina Tropical., 47(5): 547-551.

Gowdaiah PK, Reddy SS, Joseph J. 2015. Clinical study of lipid abnormalities in anti-retroviral treatment-naive HIV patients. International Journal of Advances in Medicine., 2(4): 365-369.

Habtamu WB, Mehidi KA, Tilahun YS, Yaregal AA, Bimerew LG. 2014. Prevalence and predictors of dyslipidemia on HAART and HAART naive HIV positive persons in Defense Hospital, Addis Ababa, Ethiopia. American Journal of Health Research, 2(5): 303-309.

Hejazi N, Rajikan R, Choong C, Sahar S. 2013. Metabolic abnormalities in adult HIV infected population on antiretroviral medication in Malaysia: a cross-sectional survey. BMC Public Health, 13: 758-768.

Ijeh II, Ejiké CE, Okorie U. 2010. Serum lipid profile and lipid pro-atherogenic indices of a cohort of Nigerian adults with varying glycemic and blood pressure phenotypes. Int. J. Biol. Sci., 4(6): 21022112.

Indumati V, Vijay V, Shekhanawar MS, Amareshwaras M, Shantala D. 2014. Comparison of Serum Lipid Profile in HIV Positive Patients on ART with ART Naïve Patients. Journal of Clinical and Diagnostic Research, 8(10): 6-9.

Kouakou-Siransy G, Horo K, Effo K, Koua L, Die Kakou H. Aka-Denguy E. 2015. Biological and clinical abnormalities leading to cardiovascular disease during antiretroviral treatment in a university hospital in Abidjan. Int. J. Biol. Sci., 9(4): 18351843. 
Lake JE, Currier JS. 2013. Metabolic disease in HIV infection. Lancet Infect Dis., 13: 964-975.

Lozès E, Ahoussinou C, Agassounon Tchibozo Djikpo M, Dahouégnon E, Ahossouhé N, Acoty A, de Souza C. 2012. Variabilité du taux des lymphocytes CD4 et de la charge virale chez les personnes vivant avec le VIH sous traitement antirétroviral : cas de l'hôpital saint Jean De Dieu de Tanguiéta (Bénin). Int. J. Biol. Chem. Sci., 6(2): 650-656.

Marino RB, Kingsley LA, Hussain SK, Bream JH, Penogonda S, Duggal P, Martinson J. 2015. Lipid levels in HIV positive men receiving antiretroviral therapy are not associated with copy number variation of reverse cholesterol transport pathway genes. BMC Res. Notes, 8: 697.

Nsagha DS, Weledji EP, Assob NJ, Njunda LA, Tanue EA, Kibu OD, Ayima CW, Ngowe MN. 2015. Highly active antiretroviral therapy and dyslipidemia in people living with HIV/AIDS in Fako Division, South West Region of Cameroon. BMC Cardiovascular Disorders, 15: 95.

Ojong WE, Achidi EA, Apinjoh TO, Kamga H, Njunda A. 2016. A study of the effects of HIV infection and ART treatment on the serum lipid profile of
HIV/AIDS patients attending the Nylon District Hospital, Douala, Cameroon. International Journal of Medical Science and Public Health, 5(3): 534-538.

Shahbaz S, Manicardi M, Guaraldi G, Raggi P. 2015. Cardiovascular disease in human immunodeficiency virus infected patients: A true or perceived risk? World J. Cardiol., 7(10): 633-644.

Shen Y, Wang J, Wang Z, Qi T, Song W, Tang Y, Liu L, Zhang R, Lu H. 2015. Prevalence of Dyslipidemia Among Antiretroviral-Naive HIV-Infected Individuals in China. Medecine, 94(48): $1-8$.

Tanjong RE, Teyim P, Kamga HL, Néba ES, Nkuo-Akenji T. 2016. Sero-prevalence of Human Immunodeficiency Virus and hepatitis viruses and their correlation with CD4 T-cell lymphocyte counts in pregnant women in the Buea Health District of Cameroon. Int. J. Biol. Chem. Sci., 10(1): 219-231.

Zhou DT, Kodogo V, Chokuona KF, Gomo E, Oektedalen O, Stray-Pedersen B. 2015. Dyslipidemia and cardiovascular disease risk profiles of patients attending an HIV treatment clinic in Harare, Zimbabwe. HIV/AIDS - Research and Palliative Care, 7: 145-155. 